\title{
Brane worlds at the LHC: Branons and KK gravitons
}

\author{
Jose A. R. Cembranos, Rafael L. Delgado, and Antonio Dobado \\ Departamento de Física Teórica I, Universidad Complutense de Madrid, 28040 Madrid, Spain
}

(Received 9 July 2013; published 29 October 2013)

\begin{abstract}
We study the possibility of testing some generic properties of brane world scenarios at the LHC. In particular, we pay attention to Kaluza-Klein graviton and branon production. Both signals can be dominant depending on the value of the brane tension. We analyze the differences between these two signatures. Finally, we use recent data in the single photon channel from the ATLAS Collaboration to constrain the parameter space of both phenomenologies.
\end{abstract}

DOI: 10.1103/PhysRevD.88.075021

PACS numbers: 11.25.Mj, 11.10.Lm, 11.15.Ex

\section{INTRODUCTION}

All observations carried out so far confirm the fact that there are three spatial dimensions. There is no experimental evidence that points to the existence of additional dimensions. However, there are numerous extensions of the Standard Model (SM) of particles that postulate the existence of such dimensions due to different theoretical reasons, such as supergravity or string theory (read [1] for different reviews of the subject). In the end of the past century, it was suggested that in particular constructions associated with these models, the SM particles could be understood as confined fields into three spatial dimensional manifolds or branes. On the contrary, the gravitational interaction has access to the total or bulk space. In this scenario, the fundamental scale of gravitation is not the Planck scale $M_{P}$, but another different scale $M_{D}$ that can be much lower [2,3].

This proposal, known in the literature as brane world, opened a new range of theoretical approaches and experimental possibilities to test the existence of new spatial dimensions. On the one hand, the size of the extra dimensions is much less constrained than in the old Kaluza-Klein (KK) theories. On the other hand, the lower gravitational fundamental scale allows us to analyze the hierarchy problem from a completely different perspective. Finally, the aspect that has made these attractive models and explains the large number of papers that have appeared in the last years is the rich phenomenology presented in accessible sensitivity ranges to present or future experiments.

The existence of extra dimensions leads to new degrees of freedom. The propagating gravitons along the additional space develop a tower of KK excitations from the fourdimensional point of view. On the other hand, the presence of the brane gives rise to the existence of another type of field. These models predict the existence of branons, particles associated with fluctuations of the brane in the extra dimensions. The phenomenology associated with these two types of new particles has been studied in different works. Specifically, these studies have focused on potential signatures at particle accelerators (through real [4] or virtual [5] processes), astrophysical [6,7] and cosmological
[8] observations. The study of KK gravitons allows us to constrain the number and size of extra dimensions under different assumptions. The analysis of branons restricts fundamental features of a brane (such as its tension) and local properties of the bulk space.

\section{SETTINGS OF THE BRANE WORLD SCENARIO}

The study of gravitational phenomena at the LHC is well established under the assumption of extra dimensions. In particular, one of the most popular possibilities is the socalled brane world scenario. The original idea was proposed by Rubakov and Shaposhnikov [2], but more recently Arkani-Hamed et al. and Antoniadis et al. [3] introduced the so-called Arkani-Hamed-Dimopoulos-Dvali (ADD) scenario where the SM fields (or any suitable extension of it) are confined (through some unspecified mechanism) to live in a three-dimensional brane (the world brane) while the gravitational field lives in the whole $D$-dimensional bulk space. The extra dimensions are assumed to be compact and the world brane has a tension $\tau=f^{4}$ ( $f$ is the brane tension parameter). Its thickness depends on the underlying physics producing the brane but at relatively low energies it can be safely neglected. Thus the main idea of the brane world scenario is to assume that our usual $(1+3)$-dimensional world is some sort of three-dimensional object (the brane) living in a higher $D$-dimensional bulk space $\mathcal{M}_{D}$ with $d$ additional spacial dimensions so that $D=4+d$.

In order to introduce some important concepts that we will be using later, we will split the $D$ manifold as

$$
\mathcal{M}_{D}=\mathcal{M}_{4} \times K_{d},
$$

where $\mathcal{M}_{D}$ is called the bulk space and $\mathcal{M}_{4}$ is the standard $(1+3)$-dimensional space-time brane manifold. In particular, we can take $\mathcal{M}_{4}$ to be the four-dimensional Minkowski space. The extra dimension space $K_{d}$ will be assumed to be compact, which in particular means that it is a finite volume manifold. Now we introduce the coordinates $X^{M}=\left(x^{\mu}, y^{m}\right)$ where $x^{\mu}$ parametrizes $\mathcal{M}_{4}(\mu=0$, $1,2,3)$ and $y^{m}$ parametrizes $K_{d}(m=1,2, \ldots, d)$. Also we choose the bulk-space metric $G_{M N}$ with signature $(+,-,-,-\ldots)$ so that 
$d s^{2}=G_{M N} d X^{M} d X^{N}=g_{\mu \nu} d x^{\mu} d x^{\nu}-\gamma_{m n} d y^{m} d y^{n}$,

where the metric $\gamma$ is positive definite and, according to the compactness of $K_{d}$,

$$
V_{K}=V\left(K_{d}\right)=\int d^{d} y \sqrt{|\gamma|}<\infty .
$$

For simplicity, we now consider a free real scalar field $\Phi$ of mass $M$ propagating in the bulk with action

$$
S[\Phi, G]=\int_{\mathcal{M}_{D}} d^{D} X \sqrt{|G|}\left[\frac{1}{2}\left(\partial_{M} \Phi\right)^{2}-\frac{1}{2} M^{2} \Phi^{2}\right] .
$$

The corresponding Euler-Lagrangian (Klein-Gordon) equation is

$$
\left(-\square_{D}-M^{2}\right) \Phi=0,
$$

where the $D$-dimensional d'Alambert operator is defined as

$$
\square_{D} \Phi=\nabla_{M} \nabla^{M} \Phi=\frac{1}{\sqrt{|G|}} \partial^{M}\left(\sqrt{|G|} \partial_{M} \Phi\right)
$$

The d'Alambert operator can be written as

$$
\square_{D}=\square_{4}-\square_{d},
$$

where obviously $\square_{d}$ is the d'Alambert operator on $K_{d}$ and it is positive (i.e. it has positive eigenvalues). Introducing a complete set of orthonormal functions $Y_{n}=Y_{n}(y)$ on $K_{d}$, which are solutions of the Klein-Gordon equation

$$
\square_{d} Y_{n}(y)=\lambda_{n} Y_{n}(y),
$$

we have that, due to the compactness of $K_{d}$, the spectrum $\lambda_{n}$ is discrete. In addition, we can normalize the $Y_{n}(y)$ functions so that

$$
\int_{\mathcal{K}_{d}} d^{d} y \sqrt{|\gamma|} Y_{n}(y)^{*} Y_{m}(y)=\delta_{n m} .
$$

Probably the simplest example of this setting is the $d$-dimensional torus $K_{d}=T_{d}=S_{1} \times S_{1} \times \cdots \times S_{1} \quad(d$ times). On each circle $S_{1}$ we introduce the coordinate $y^{m}$ which clearly is periodic in the sense that $y^{m}$ and $y^{m}+2 \pi R$ represent the same point ( $R$ is the common radius of the circles). Thus we can consider only $y^{m}$ values lying in the interval $y^{m} \in[0,2 \pi R]$. The volume of the torus is $V_{T}=$ $V\left(T_{d}\right)=(2 \pi R)^{d}$ and $\square_{d}=\nabla_{d}^{2}$ is just the $d$-dimensional Laplace operator. Its eigenvalues $\lambda_{n}$ can be labeled by a set of $d$ integers $n=\left(n_{1}, n_{2}, \ldots, n_{d}\right)$ and they can be easily found to be

$$
\lambda_{n}=\lambda_{\left(n_{1}, n_{2}, \ldots, n_{d}\right)}=\sum_{m=1}^{d} \frac{n_{m}^{2}}{R^{2}},
$$

and the corresponding eigenfunctions are

$$
Y_{\left(n_{1}, n_{2}, \ldots, n_{d}\right)}(y)=\frac{1}{\sqrt{V_{T}}} \exp \left\{\frac{i \sum_{m=1}^{d} n_{m} y^{m}}{R}\right\} .
$$

Notice that $\lambda_{0}=0\left(Y_{0}=1 / \sqrt{V_{T}}\right)$ but, for $n \neq 0, \lambda_{n}$ goes as $1 / R^{2}$.

In the general case we can expand the bulk field $\Phi$ in terms of the $Y_{n}(y)$ as follows:

$$
\Phi(X)=\Phi(x, y)=\sum_{n} \phi_{n}(x) Y_{n}(y) .
$$

This is the so-called Kaluza-Klein expansion, and plugging it into the $\Phi$ action one gets

$$
\begin{aligned}
S[\Phi, G]= & \int_{M_{D}} d^{4} x \sqrt{|g|}\left\{\phi_{0}\left(-\square_{4}-M^{2}\right) \phi_{0}\right. \\
& \left.+\sum_{n \neq 0} \phi_{n}^{*}\left(-\square_{4}-M_{n}^{2}\right) \phi\right\},
\end{aligned}
$$

where

$$
M_{n}^{2}=M^{2}+\lambda_{n}
$$

For the case of the torus we have

$\Phi(x, y)=\frac{1}{(2 \pi R)^{d / 2}} \sum_{\left(n_{1}, n_{2}, \ldots, n_{d}\right)} \phi_{\left(n_{1}, n_{2}, \ldots, n_{d}\right)}(x) \exp \left\{\frac{i \sum_{m=1}^{d} n_{m} y^{m}}{R}\right\}$,

where $\phi_{\left(n_{1}, n_{2}, \ldots, n_{d}\right)}(x)$ are the KK modes for the $\Phi$ field. They can be considered $M_{4}$ fields with masses:

$$
M_{\left(n_{1}, n_{2}, \ldots, n_{d}\right)}^{2}=M^{2}+\frac{\sum_{m=1}^{d} n_{m}^{2}}{R^{2}} .
$$

For the simple case $d=1$ and $M=0, \phi_{0}$ becomes a real zero mode. Notice also that for $n \neq 0$ the KK modes are complex and massive even when $M^{2}=0$ (no mass term on the bulk for the $\Phi$ field). Also since $\Phi$ is a real field as $Y_{n}^{*}(y)=Y_{-n}(y)$ we have $\phi_{n}^{*}(y)=\phi_{-n}(y)$. Thus the complex conjugate of some KK mode represents the same mode propagating in the opposite direction of the $T_{d}$ internal space.

In the general case a real bulk scalar field $\Phi(X)$ is equivalent to a $\mathrm{KK}$ tower of massive complex $M_{4}$ fields $\phi_{n}(x)$ with masses $M_{n}^{2}=M^{2}+\lambda_{n}$. At low energies $E \ll$ $1 / R$ ( $R$ being the typical size of the extra dimensions i.e. $V_{K} \sim R^{d}$ ) only the real zero mode survives. However, at higher energies more and more KK modes become relevant and must be taken into account.

Note that even if the size of the extra dimensions is too small to be directly observable, their existence could be probed by detecting these KK modes of the effective fourdimensional theory. The spectrum of the KK tower will give us information about the geometry of the internal space. At low energies only zero modes can be excited (dimensional reduction).

\section{GRAVITONS}

According to the general idea that our universe is a 3-brane, i.e. a three-dimensional smooth object living in 
a higher dimensional space (the $D$-dimensional bulk space) and using the notation introduced above it is very easy to find that

$$
M_{P}^{2}=M_{4}^{2}=V\left(K_{d}\right) M_{D}^{D-2},
$$

where $M_{D}$ is the $D$-dimensional Planck scale defined. Thus, the Einstein-Hilbert action is given by

$$
S_{\mathrm{EH}}=\frac{M_{D}^{D-2}}{16 \pi} \int_{\mathcal{M}_{D}} d^{D} X \sqrt{|G|}\left[R_{D}-(D-2) \Lambda_{D}\right],
$$

where we have introduced the bulk cosmological constant $\Lambda_{D}$. Obviously, $M_{P}=M_{4}$ (we are using $\hbar=c=1$ units), so that $M_{P} \simeq 1.2 \times 10^{19} \mathrm{GeV}$.

One of the important points of the original idea of the ADD scenario is to have $R$ large enough so that the $D$-dimensional Planck mass $M_{D}$ (the fundamental scale of gravity) could be of the order of the $\mathrm{TeV}$ scale, thus solving, or at least putting in a completely new setting, the hierarchy problem since for $v \simeq 250 \mathrm{GeV}$ being the electroweak symmetry breaking scale we could have

$$
M_{D} \sim 4 \pi v \ll M_{P} .
$$

Therefore, the huge hierarchy is produced by the large volume of the extra dimension space $K_{d}$. For example for $d=1, M_{D} \sim 1 \mathrm{TeV}$ requires $R \sim 10^{13} \mathrm{~cm}$, which is ruled out by our knowledge of the Newton law at the Solar System scale. For $d=2, R \sim 0.1 \mathrm{~mm}$, which is close to the experimental limit coming from the study of possible deviations from the Newton law at the sub-millimeter scale. For $d \geq 3, R$ must be of the order or smaller than $10^{-7} \mathrm{~cm}$, which in principle is well below any experimental constraint.

The simplest action describing the ADD model is

$$
\begin{aligned}
S_{\mathrm{ADD}}= & \frac{M_{D}^{D-2}}{16 \pi} \int_{\mathcal{M}_{D}} d^{D} X \sqrt{|G|}\left[R_{D}-(D-2) \Lambda_{D}\right] \\
& +\int_{\mathcal{M}_{4}} d^{4} x \sqrt{|g|}\left(\mathcal{L}_{\mathrm{SM}}(g, \Phi)-\tau\right),
\end{aligned}
$$

where $\mathcal{M}_{4}$ is the brane world sheet with coordinates $x^{\mu}$, $\mathcal{L}_{\text {SM }}$ is the SM Lagrangian defined on $\mathcal{M}_{4}, \Phi$ represents all the SM fields and the last term is just the Nambu-Goto action for the brane. Any point on $\mathcal{M}_{4}$ will have bulk coordinates $Y^{M}=Y^{M}(x)$. Then the interval on this fourdimensional manifold is given by

$$
\begin{aligned}
d s^{2} & =G_{M N} d Y^{M} d Y^{N}=G_{M N} \frac{\partial Y^{M}}{\partial x^{\mu}} \frac{\partial Y^{N}}{\partial x^{\nu}} d x^{\mu} d x^{\nu} \\
& \equiv g_{\mu \nu} d x^{\mu} d x^{\nu} .
\end{aligned}
$$

The $g_{\mu \nu}$ metric defined on $\mathcal{M}_{4}$ is called the induced metric (or the $G_{M N}$ pullback on $\mathcal{M}_{4}$ ). It contains not only the geometrical properties associated with $\mathcal{M}_{D}$ but also the ones related to the way $\mathcal{M}_{4}$ is embedded in $\mathcal{M}_{D}$.
In order to study some of the properties of this model, and for another reason that will be explained at the end of this section, we will concentrate mainly on the simple case $\mathcal{M}_{D}=M_{4} \times T_{d}$. Notice that in particular this means that we are neglecting any brane fluctuation so this can be understood as a case of having the brane tension scale $f$ much larger than the other relevant scales in the system. In the next section we will consider the effects produced by these brane fluctuations for lower values of the tension parameter $f$ (flexible brane case).

Now, in order to study the graviton excitations, we write the bulk metric as

$$
G_{M N}(x, y)=\eta_{M N}+\frac{2}{\bar{M}_{D}^{1+d / 2}} h_{M N}(x, y),
$$

where $\bar{M}_{D}^{D-2} \equiv M_{D}^{D-2} / 4 \pi$ is the reduced fundamental scale and $h_{M N}(x, y)$ is the bulk graviton field. The normalization is chosen so that the corresponding action at the lowest order is the canonical one

$$
\begin{aligned}
S[h]= & \int_{\mathcal{M}_{D}} d^{D} X\left[\frac{1}{4} \partial^{R} h^{M N} \partial_{R} h_{M N}-\frac{1}{2} \partial^{R} h^{M N} \partial_{M} h_{R N}\right. \\
& \left.+\frac{1}{2} \partial^{M} h \partial^{L} h_{L M}-\frac{1}{4} \partial^{M} h \partial_{M} h\right]
\end{aligned}
$$

where $h=\eta_{M N} h^{M N}$. The graviton field can be KK expanded as

$$
\begin{aligned}
h_{M N}(x, y)= & \frac{1}{(2 \pi R)^{d / 2}} \sum_{\left(n_{1}, n_{2}, \ldots, n_{d}\right)} h_{M N}^{\left(n_{1}, n_{2}, \ldots, n_{d}\right)}(x) \\
& \times \exp \left\{\frac{i \sum_{m=1}^{d} n_{m} y^{m}}{R}\right\},
\end{aligned}
$$

where $h_{M N}^{\left(n_{1}, n_{2}, \ldots, n_{d}\right)}(x)$ are the KK modes for the graviton field with masses

$$
M_{\left(n_{1}, n_{2}, \ldots, n_{d}\right)}^{2}=\frac{\sum_{m=1}^{d} n_{m}^{2}}{R^{2}} .
$$

Therefore, in addition to the usual massless graviton, we will have an infinite tower of complex massive gravitons. One important observation here is that the gap or mass distance between two consecutive massive gravitons goes as $\triangle M \sim 1 / R$. This means that for large enough extra dimensions the KK graviton spectrum can be considered as almost continuous. As we will see later this is an important fact that opens the possibility of producing gravitons in a detectable rate under some conditions.

Thus, the massless zero mode graviton $h_{\mu \nu}(x)$ has a whole tower of massive KK partners, $h_{\mu \nu}^{n}(x)$, which are massive $J=2$ fields with five physical polarization states. The additional degrees of freedom come from a sort of Higgs mechanism, present in Kaluza-Klein theories, where the field $h_{\mu \nu}^{n}(x)$ eats some of the extra dimensional excitations producing the tower of massive $J=2 \mathrm{KK}$ modes. The effective Lagrangian describing the free evolution of 
these massive fields can be taken to be the well-known Fierz-Pauli action

$$
\begin{aligned}
S_{\mathrm{FP}}[h]= & \sum_{n} \int_{M_{4}} d^{4} x\left[\frac{1}{4} \partial^{\rho} h^{\mu \nu(n)} \partial_{\rho} h_{\mu \nu}^{(n)}-\frac{1}{2} \partial^{\rho} h^{\mu \nu(n)} \partial_{\mu} h_{\rho \nu}^{(n)}\right. \\
& +\frac{1}{2} \partial^{\mu} h^{(n)} \partial^{\lambda} h_{\lambda \mu}^{(n)}-\frac{1}{4} \partial^{\mu} h^{(n)} \partial_{\mu} h^{(n)} \\
& \left.-\frac{1}{4} M_{n}^{2}\left(h^{\mu \nu(n)} h_{\mu \nu}^{(n)}-\left(h^{(n)}\right)^{2}\right)\right],
\end{aligned}
$$

where, for example, $n$ should be understood as $n=$ $\left(n_{1}, n_{2}, \ldots, n_{d}\right)$ in the torus case. In particular, $M_{n}^{2}=$ $M_{\left(n_{1}, n_{2}, \ldots, n_{d}\right)}^{2}$. The above action naturally leads to the set of equations

$$
h^{(n)}=0 \quad \partial_{\mu} h^{\mu \nu(n)}=0 \quad\left(\square+M_{n}^{2}\right) h^{\mu \nu(n)}=0 .
$$

Here the first two equations are the five constraints that reduce the original degrees of freedom of the symmetric tensors $h^{\mu \nu(n)}$ from ten to five and the last one is just the Klein-Gordon equation expected for free massive bosons. Now, in order to study the interaction between massive gravitons and the SM particles we start from the SM piece of the ADD action

$$
S_{\mathrm{SM}}[g, \Phi]=\int_{\mathcal{M}_{4}} d^{4} x \sqrt{|g|} \mathcal{L}_{\mathrm{SM}}(g, \Phi),
$$

and then we expand it around the $\eta_{\mu \nu}$ Minkowskian $\left(M_{4}\right)$ brane metrics

$$
\begin{aligned}
S_{\mathrm{SM}}[g, \Phi]= & \int_{M_{4}} d^{4} x \mathcal{L}_{\mathrm{SM}}(\eta, \Phi) \\
& +\left.\int_{M_{4}} d^{4} x \frac{\delta S_{\mathrm{SM}}}{\delta g_{\mu \nu}(x)}\right|_{g=\eta} \delta g_{\mu \nu}(x)+\cdots
\end{aligned}
$$

But in our setting we have

$$
\delta g_{\mu \nu}(x)=\frac{2}{\bar{M}_{D}^{1+d / 2}} h_{\mu \nu}(x),
$$

and the SM energy momentum tensor is

$$
T_{\mathrm{SM}}^{\mu \nu}=-\left.\frac{2}{\sqrt{|g|}} \frac{\delta S_{\mathrm{SM}}}{\delta g_{\mu \nu}(x)}\right|_{g=\eta} .
$$

Therefore, the $S_{\mathrm{SM}}$ action can be split as the usual SM action in flat space-time $M_{4}$ plus an interacting term $S_{\text {int }}$ given by

$$
S_{\text {int }}[h, \Phi]=-\frac{1}{\bar{M}_{D}^{1+d / 2}} \int_{M_{4}} d^{4} x T_{\mathrm{SM}}^{\mu \nu} h_{\mu \nu}
$$

We can use the relation between the $D$-dimensional fundamental scale of gravity $M_{D}$ and the Planck scale $M_{P}$, to write the interaction of the KK mode expansion for the graviton field in the following way:

$S_{\text {int }}[h, \Phi]=-\frac{1}{\bar{M}_{P}} \sum_{\left(n_{1}, n_{2}, \ldots, n_{d}\right)} \int_{M_{4}} d^{4} x T_{\mathrm{SM}}^{\mu \nu} h_{\mu \nu}^{\left(n_{1}, n_{2}, \ldots, n_{d}\right)}$,
As it is expected, the SM and graviton interactions are suppressed by the Planck mass.

From this action, following the standard procedure, it is possible to obtain the Feynman rules for the different couplings such as graviton-fermion antifermion, gravitonphoton-photon, graviton-photon-fermion-antifermion, graviton-gluon-gluon-gluon and many others. Some attention must be paid to the gauge fixing conditions for the graviton field, which should give rise to the appropriate propagators and polarization wave functions, that must reproduce the two polarization states of the massless graviton and the five polarization states of the massive gravitons. In the case of virtual gravitons one should also pay attention to the corresponding ghost fields.

Thus, it is possible for instance to compute the amplitude of the process $e^{+} e^{-} \rightarrow \gamma h^{n}$. The signal for this reaction would be very clear since gravitons escape from detection and then we are left just with one single photon event with missing energy and $P_{T}$. Nevertheless the cross section for producing one graviton is strongly suppressed by the Planck mass and one expects

$$
\sigma \sim \frac{1}{M_{P}^{2}} .
$$

However, if one considers the cross section for producing any KK graviton, things are completely different. As it was commented above for large $R$, the KK spectrum can be considered continuous. Let us define $N(k)$ as the number of KK modes with modulus $|\vec{k}|$ of the extra dimension momentum $\vec{k}=\left(k_{1}, \ldots, k_{d}\right)$ lesser than or equal to $k$. Then, it is easy to see that

$$
d N \sim R^{d} S_{d-1} k^{d-1} d k,
$$

where

$$
S_{n}=\frac{(2 \pi)^{n / 2}}{\Gamma(n / 2)} .
$$

Therefore, for some given energy $E$, the number of available KK gravitons is

$$
N(E)=\int_{0}^{E} n\left(E^{\prime}\right) d E^{\prime} \sim \frac{S_{d-1} M_{P}^{2} E^{d}}{d M_{D}^{d+2}},
$$

where $n(E)=d N / d E$ is the energy density of KK states energy density. Thus, we finally arrive at the conclusion that the cross section for producing any KK graviton goes as [9]

$$
\sigma \sim \frac{S_{d-1}}{d} \frac{E^{d}}{M_{D}^{d+2}}
$$

and therefore it is not suppressed by the Planck mass but by the fundamental scale $M_{D}$ which in this framework is supposed to be of the order of the TeV. This is a very interesting property of the ADD model since it opens the possibility of having detectable gravitational interactions at the LHC.

From this example based in the case $K_{d}=T_{d}$ we see that the total cross section for producing gravitons in the 
large $R$ limit (which means continuous spectrum) depends on the KK state density $n(E)$ i.e. on the spectrum of the KK gravitons. Thus in principle by measuring carefully the cross section for graviton production, let us say, at the LHC, it could be possible to obtain information about the spectrum which in turn could carry information about the $K_{d}$ (extra dimension space) geometry and topology. We can illustrate this idea by comparison between the two simple $d=2$ cases $K_{2}=T^{2}$ and $K_{2}=S^{2}$. As discussed above the graviton spectrum for the first case is given by

$$
M_{\left(n_{1}, n_{2}\right)}^{2}=\frac{1}{R^{2}}\left(n_{1}^{2}+n_{2}^{2}\right) .
$$

In the $S^{2}$ case the volume of the extra dimension space is given by $V\left(S^{2}\right)=4 \pi R^{2}$ where $R$ is the sphere radius (note the different geometrical meaning of $R$ in both cases). The graviton field can be KK expanded as

$$
h_{M N}(x, y)=\frac{1}{R} \sum_{l, m} h_{M N}^{l m}(x) Y_{l m}(y),
$$

where $Y_{l m}(y)$ with $y=(\theta, \phi)$ are the standard spherical harmonics and thus $l=0,1,2 \ldots$ and $m=-l, \ldots 0, \ldots l$ and the corresponding spectrum is

$$
M_{l m}^{2}=\frac{1}{R^{2}} l(l+1) .
$$

Therefore, as expected, the two spectra are different having different gaps and degeneracies. In the large $R$ limit we can obtain the state densities. According to our previous discussion for the $T^{2}$ case, we get

$$
n_{T^{2}}(E)=\frac{\pi}{2} R^{2} E
$$

For the $S^{2}$ case it is not difficult to find

$$
n_{S^{2}}(E)=2 R^{2} E \text {. }
$$

This example shows that in the continuous limit, both the gap and degeneracy information contained in the discrete spectra are washed out, making the two cases indistinguishable, at least from the practical point of view, as they concern the graviton production cross sections. For this reason, we will concentrate on the torus case, as representative of many other possible compactified extra dimension spaces.

\section{BRANE FLUCTUATIONS (BRANONS)}

In this section we consider branons [10,11], another kind of excitation that could possibly be present in the brane world scenarios which are particularly interesting when the scale tension parameter $f$ is low enough, i.e. in the case of a flexible brane. As in the previous discussion the brane lies along $\mathcal{M}_{4}$ but to start with we will neglect the gravitons (see next section). The bulk space $\mathcal{M}_{D}$ metrics will be assumed to have the general form $[11,12]$

$$
G_{M N}=\left(\begin{array}{cc}
\tilde{g}_{\mu \nu}(x) & 0 \\
0 & -\tilde{g}_{m n}^{\prime}(y)
\end{array}\right) \text {. }
$$

The position of the brane in the bulk can be parametrized as $Y^{M}=\left(x^{\mu}, Y^{m}(x)\right)$ where we have chosen the bulk coordinates so that the first four are identified with the space-time brane coordinates $x^{\mu}$. We assume the brane to be created at a certain point in $K_{d}$, i.e. $Y^{m}(x)=Y_{0}^{m}$, which corresponds to its ground state. The induced metric on the brane in this particular case is given by $g_{\mu \nu}=\tilde{g}_{\mu \nu}=G_{\mu \nu}$. However, when brane excitations are present, the induced metric is given by

$$
\begin{aligned}
g_{\mu \nu} & =\partial_{\mu} Y^{M} \partial_{\nu} Y^{N} G_{M N}(x, Y(x)) \\
& =\tilde{g}_{\mu \nu}(x, Y(x))-\partial_{\mu} Y^{m} \partial_{\nu} Y^{n} \tilde{g}_{m n}^{\prime}(Y(x)) .
\end{aligned}
$$

Since the mechanism responsible for the creation of the brane is in principle unknown, we will assume that the brane dynamics can be described by an effective action. At low energies the dominant term is the one having the appropriate symmetries, with the least possible number of derivatives of the induced metric. This principle leads us to

$$
S_{B}=-f^{4} \int_{M_{4}} d^{4} x \sqrt{g}
$$

where $d^{4} x \sqrt{g}$ is the volume element of the brane. Notice that this lowest order term is the Nambu-Goto action introduced below.

In the absence of the 3-brane, the metric (44) possesses an isometry group which we will assume to be of the form $G\left(\mathcal{M}_{D}\right)=G\left(\mathcal{M}_{4}\right) \times G\left(K_{d}\right)$. The presence of the brane will break spontaneously all the $K_{d}$ isometries, except those that leave the point $Y_{0}$ (the brane ground state) unchanged. The group $G\left(K_{d}\right)$ is spontaneously broken down to $H\left(Y_{0}\right)$, where $H\left(Y_{0}\right)$ denotes the isotropy group (or little group) of the point $Y_{0}$ and we can define the coset space $K=G\left(\mathcal{M}_{D}\right) /\left(G\left(\mathcal{M}_{4}\right) \times H\left(Y_{0}\right)\right)=G\left(K_{d}\right) / H\left(Y_{0}\right)$.

When the $K_{d}$ space is homogeneous the little group $H\left(Y_{0}\right)$ is $Y_{0}$ independent and $H\left(Y_{0}\right) \equiv H$. The coset $K$ is isomorphic to $K_{d}$ and the isometries are just translations. In this case the branon fields $(\pi)$, defined as Gaussian coordinates on the coset $K$, can be identified, with properly chosen coordinates in the extra space $K_{d}$, as for example

$$
\pi^{\alpha}=f^{2} \delta_{m}^{\alpha} y^{m} .
$$

In the following, for the sake of simplicity, we will consider only $K_{d}$ homogeneous spaces.

According to the previous discussion, we can write the induced metric on the brane in terms of branon fields as

$$
g_{\mu \nu}=\tilde{g}_{\mu \nu}(x)-\tilde{g}_{m n}^{\prime} \frac{\partial Y^{m}}{\partial \pi^{\alpha}} \frac{\partial Y^{n}}{\partial \pi^{\beta}} \partial_{\mu} \pi^{\alpha} \partial_{\nu} \pi^{\beta} .
$$


Introducing the metrics $h_{\alpha \beta}(\pi)$ as

$$
h_{\alpha \beta}(\pi)=f^{4} \tilde{g}_{m n}^{\prime}(Y(\pi)) \frac{\partial Y^{m}}{\partial \pi^{\alpha}} \frac{\partial Y^{n}}{\partial \pi^{\beta}},
$$

we have

$$
g_{\mu \nu}=\tilde{g}_{\mu \nu}(x)-\frac{1}{f^{4}} h_{\alpha \beta}(\pi) \partial_{\mu} \pi^{\alpha} \partial_{\nu} \pi^{\beta} .
$$

The above scheme leading to massless branons is only valid if the isometry pattern introduced before is exact. However, in more general situations, these symmetries are only approximately realized and branons will acquire mass $[12,13]$. In order to illustrate how this could happen explicitly, let us perturb the four-dimensional components of the background metric and let $\tilde{g}_{\mu \nu}$ be dependent, not only on the $x$ coordinates, but also on the $y$ ones $[12,13]$ :

$$
G_{M N}=\left(\begin{array}{cc}
\tilde{g}_{\mu \nu}(x, y) & 0 \\
0 & -\tilde{g}_{m n}^{\prime}(y)
\end{array}\right) .
$$

This has to be done in such a way that the $G\left(K_{d}\right)$ piece of the full isometry group is explicitly broken. Notice that the breaking of the $G\left(K_{d}\right)$ group by perturbing only the internal metric $\tilde{g}_{m n}^{\prime}(y)$ does not lead to a mass term for the branons.

In order to calculate the branon mass matrix, we need to know first the ground state around which the brane is fluctuating. With that purpose, we will consider for simplicity the lowest-order action, given by

$$
S_{\mathrm{eff}}^{(0)}[\pi]=-f^{4} \int_{M_{4}} d^{4} x \sqrt{\tilde{g}(x, Y(x))},
$$

which will have an extreme provided by

$$
\begin{aligned}
\delta S_{\mathrm{eff}}^{(0)}[\pi]=0 & \Rightarrow \delta \sqrt{\tilde{g}}=\frac{1}{2} \sqrt{\tilde{g}} \tilde{g}^{\mu \nu} \delta \tilde{g}_{\mu \nu} \\
=0 & \Rightarrow \tilde{g}^{\mu \nu} \partial_{m} \tilde{g}_{\mu \nu}=0 .
\end{aligned}
$$

This is a set of equations whose solution $Y_{0}^{m}(x)$ determines the shape of the brane in its ground state for a given background metric $\tilde{g}_{\mu \nu}$. In addition, the condition for the energy to be minimum requires

$$
\left.\frac{\delta^{2} S_{\mathrm{eff}}^{(0)}}{\delta Y^{m} \delta Y^{n}}\right|_{Y=Y_{0}}<0
$$

which means

$$
\frac{f^{4}}{4} \sqrt{\tilde{g}} \tilde{g}^{\mu \nu}\left(\partial_{n} \partial_{m} \tilde{g}_{\mu \nu}-2 \tilde{g}^{\rho \sigma} \partial_{n} \tilde{g}_{\nu \sigma} \partial_{m} \tilde{g}_{\mu \rho}\right)>0 ;
$$

i.e., the eigenvalues of the above matrix should be positive. This implies that the action should have a minimum for static configurations.

In order to obtain the explicit expression of the branon mass matrix, we expand $\tilde{g}_{\mu \nu}(x, y)$ around $y^{m}=Y_{0}^{m}$ in terms of the $\pi^{\alpha}$ fields:

$$
\begin{aligned}
\tilde{g}_{\mu \nu}(x, y)= & \tilde{g}_{\mu \nu}\left(x, Y_{0}\right)+\partial_{m} \tilde{g}_{\mu \nu}\left(x, Y_{0}\right)\left(Y^{m}-Y_{0}^{m}\right) \\
& +\frac{1}{2} \partial_{m} \partial_{n} \tilde{g}_{\mu \nu}\left(x, Y_{0}\right)\left(Y^{m}-Y_{0}^{m}\right)\left(Y^{n}-Y_{0}^{n}\right)+\cdots \\
= & \tilde{g}_{\mu \nu}\left(x, Y_{0}\right)+\frac{1}{f} V_{\alpha \mu \nu}^{(1)} \pi^{\alpha}+\frac{1}{f^{2}} V_{\alpha \beta \mu \nu}^{(2)} \pi^{\alpha} \pi^{\beta}+\cdots
\end{aligned}
$$

The linear term in branon fields is written as

$$
V_{\alpha \mu \nu}^{(1)}=\left.\partial_{m} \tilde{g}_{\mu \nu}(x, y)\right|_{y=Y_{0}} \frac{\xi_{\alpha}^{m}}{k f},
$$

where $\xi_{\alpha}$ are the Killing vectors corresponding to the broken generators defining the coset $K \sim K_{d}$, i.e., those generators of $G\left(\mathcal{M}_{D}\right)=G\left(\mathcal{M}_{4}\right) \times G\left(K_{d}\right)$ not present in $H$. These Killing vectors are normalized so that $k^{2}=$ $16 \pi / M_{P}^{2}$, with $M_{P}$ the four-dimensional Planck mass.

The quadratic term takes the general form

$$
\begin{aligned}
V_{\alpha \beta \mu \nu}^{(2)}= & \left.\left.\frac{f^{2}}{2} \partial_{m} \tilde{g}_{\mu \nu}(x, y)\right|_{y=Y_{0}} \frac{\partial^{2} Y^{m}}{\partial \pi^{\alpha} \partial \pi^{\beta}}\right|_{\pi=0} \\
& +\left.\frac{1}{2} \partial_{m} \partial_{n} \tilde{g}_{\mu \nu}(x, y)\right|_{y=Y_{0}} \frac{\xi_{\alpha}^{m} \xi_{\beta}^{n}}{k^{2} f^{2}} .
\end{aligned}
$$

Here, we have used the fact that the action of an element of $G\left(K_{d}\right)$ on $K_{d}$ will map $Y_{0}$ onto some other point with coordinates

$$
\begin{aligned}
Y^{m}(x) & =Y^{m}\left(Y_{0}, \pi^{\alpha}(x)\right) \\
& =Y_{0}^{m}+\frac{1}{k f^{2}} \xi_{\alpha}^{m}\left(Y_{0}\right) \pi^{\alpha}(x)+O\left(\pi^{2}\right) .
\end{aligned}
$$

Substituting the above expression back into (50), we get the expansion of the induced metric in branon fields:

$$
\begin{aligned}
g_{\mu \nu}= & \tilde{g}_{\mu \nu}\left(x, Y_{0}\right)-\frac{1}{f^{4}} \delta_{\alpha \beta} \partial_{\mu} \pi^{\alpha} \partial_{\nu} \pi^{\beta}+\frac{1}{f} V_{\alpha \mu \nu}^{(1)} \pi^{\alpha} \\
& +\frac{1}{f^{2}} V_{\alpha \beta \mu \nu}^{(2)} \pi^{\alpha} \pi^{\beta}+O\left(\pi^{4}\right) .
\end{aligned}
$$

We have also used the fact that since $\pi^{\alpha}$ must be properly normalized scalar fields, the $Y^{m}$ coordinates should be normal and geodesic in a neighborhood of $Y_{0}^{m}$ and, in particular, they cannot be angular coordinates. This implies that we can write $h_{\alpha \beta}(\pi=0)=\delta_{\alpha \beta}$.

Assuming for concreteness that, in the ground state, the four-dimensional background metric is flat, i.e. $\tilde{g}_{\mu \nu}\left(x, Y_{0}\right)=\eta_{\mu \nu}$, the appearance of the $V_{\alpha_{1} \alpha_{2} \ldots \alpha_{i} \mu \nu}^{(i)}$ tensors in (56) could break Lorentz invariance, unless they factor out as $V_{\alpha_{1} \alpha_{2} \ldots \alpha_{i} \mu \nu}^{(i)}=M_{\alpha_{1} \alpha_{2} \ldots \alpha_{i}}^{(i)} \eta_{\mu \nu} /\left(4 f^{2}\right)$. With this assumption, the linear term $V_{\alpha \mu \nu}^{(1)}$ vanishes identically due to the condition of minimum for the brane energy (53), and the $M_{\alpha \beta}^{(2)}$ coefficient in the quadratic term can be identified with the branon mass matrix. Thus we find 


$$
\begin{aligned}
\sqrt{g}= & 1-\frac{1}{2 f^{4}} \eta^{\mu \nu} \delta_{\alpha \beta} \partial_{\mu} \pi^{\alpha} \partial_{\nu} \pi^{\beta} \\
& +\frac{1}{2 f^{4}} M_{\alpha \beta}^{(2)} \pi^{\alpha} \pi^{\beta}+\cdots
\end{aligned}
$$

Notice that this expression requires that both $\partial \pi / f^{2}$ and $M^{2} \pi^{2} / f^{4}$ be small. This includes different types of approaches, such as low-energy expansions with small branon masses compared to $f$, or low-energy expansions with possible large masses and small $\pi / f$ factors.

The different terms in the effective action can be organized according to the number of branon fields,

$$
S_{\text {eff }}[\pi]=S_{\text {eff }}^{(0)}[\pi]+S_{\text {eff }}^{(2)}[\pi]+\cdots,
$$

where the zeroth order term is just a constant. The free action contains the terms with two branons,

$S_{\mathrm{eff}}^{(2)}[\pi]=\frac{1}{2} \int_{M_{4}} d^{4} x\left(\delta_{\alpha \beta} \partial_{\mu} \pi^{\alpha} \partial^{\mu} \pi^{\beta}-M_{\alpha \beta}^{2} \pi^{\alpha} \pi^{\beta}\right)$.

In principle one can always diagonalize the squared mass matrix $M_{\alpha \beta}^{2}$ to obtain the physical branon fields with masses $M_{\alpha}$.

In order to study the possible phenomenological consequences of the brane fluctuations it is very important to obtain the coupling of branons to the SM particles. To this end it is enough to consider the case $\tilde{g}_{\mu \nu}=\eta_{\mu \nu}$. Now we can proceed as in [11], where the SM action on the brane is expanded in branon fields through the induced metric. Thus the complete action, including terms up to two branons, is given by $[12,14]$

$$
\begin{aligned}
S_{B}= & \int_{M_{4}} d^{4} x \sqrt{g}\left[-f^{4}+\mathcal{L}_{\mathrm{SM}}\right] \\
= & \int_{M_{4}} d^{4} x\left[-f^{4}+\mathcal{L}_{\mathrm{SM}}\left(\eta_{\mu \nu}\right)+\frac{1}{2} \delta_{\alpha \beta} \partial_{\mu} \pi^{\alpha} \partial^{\mu} \pi^{\beta}\right. \\
& -\frac{1}{2} M_{\alpha \beta}^{2} \pi^{\alpha} \pi^{\beta}+\frac{1}{8 f^{4}}\left(4 \delta_{\alpha \beta} \partial_{\mu} \pi^{\alpha} \partial_{\nu} \pi^{\beta}\right. \\
& \left.\left.-M_{\alpha \beta}^{2} \pi^{\alpha} \pi^{\beta} \eta_{\mu \nu}\right) T_{\mathrm{SM}}^{\mu \nu}\right]+\mathcal{O}\left(\pi^{3}\right),
\end{aligned}
$$

where $T_{\mathrm{SM}}^{\mu \nu}\left(\eta_{\mu \nu}\right)$ is the SM energy-momentum tensor as defined in the case of gravitons considered in the previous section:

$$
T_{\mathrm{SM}}^{\mu \nu}=-\left.\left(\tilde{g}^{\mu \nu} \mathcal{L}_{\mathrm{SM}}+2 \frac{\delta \mathcal{L}_{\mathrm{SM}}}{\delta \tilde{g}_{\mu \nu}}\right)\right|_{\tilde{g}_{\mu \nu}=\eta_{\mu \nu}} .
$$

Notice that no single-branon interactions, which will be related to Lorentz invariance breaking, are present in this action. In addition the quadratic expression in (64) is valid for any $K_{d}$ space, regardless of the particular form of the metric $\tilde{g}_{m n}^{\prime}$. In fact, the form of the couplings only depends on the number of branon fields, their mass and the brane tension. The dependence on the geometry of the extra dimensions will appear only at higher orders, contrary to the case of gravitons. Therefore branons interact always in pairs with the SM matter fields. In addition, due to their geometric origin, those interactions are very similar to the gravitational ones since the $\pi$ fields couple to all the matter fields through the energy-momentum tensor and with the same strength, which is suppressed by a $f^{4}$ factor. In fact, branons couple as gravitons do, with the identification $[12,15]$

$$
-\frac{1}{\bar{M}_{P}} h_{\mu \nu} \rightarrow \frac{1}{8 f^{4}}\left(4 \delta_{\alpha \beta} \partial_{\mu} \pi^{\alpha} \partial_{\nu} \pi^{\beta}-M_{\alpha \beta}^{2} \pi^{\alpha} \pi^{\beta} \eta_{\mu \nu}\right),
$$

where $h_{\mu \nu}$ is the graviton field in linearized gravity. As in the graviton case, by using standard methods, it is possible to find the relevant Feynman rules, amplitudes and cross section for producing branons (by pairs) in, for example, the LHC. From the discussion above, it is clear that these branons are in general massive and stable (at least the lightest of them) and, therefore, they would escape detection, given that their main signature is missing energy and momentum as is the case also for gravitons.

\section{COLLIDER PHENOMENOLOGY: SINGLE PHOTON CHANNEL}

From the discussion presented so far it is clear that probably the most outstanding property of flexible brane world scenarios is the presence of two types of generic excitations, namely, KK gravitons and branons. Curiously enough the experimental signatures for producing these excitations starting from SM particles in colliders as the LHC are in both cases missing energy and transverse momentum. Therefore, finding an important number of missing energy events at the LHC could suggest some chances for a brane world case (note however that other scenarios like the minimal supersymmetric SM could also produce such signals, but in that case one expects the production of many other new SUSY particles that should also be present).

The graviton production cross section is of the order $\sigma_{G} \sim(E R)^{d} / M_{P}^{2}$ and the branon production cross section goes as $\sigma_{B} \sim E^{6} / f^{8}$. Therefore it is clear that, for some given extra dimension space size $R$, the relative production rate of gravitons and branons is controlled by the brane tension parameter $f$. For rigid branes (high values of $f$ ) we expect graviton production dominance, while for flexible branes (low $f$ ) we expect branon production to be more abundant.

In this sense, both effects are complementary. The first one is important in the solid brane limit, when $M_{D} / f$ is small. The second one is dominant in the opposite limit, for 
flexible branes with a large value of $M_{D} / f$. Indeed, it has been shown in Refs. $[16,17]$ that in the flexible brane limit, the KK graviton coupling is exponentially suppressed and the KK gravitons decouple. ${ }^{1}$

In the rest of this work we will study in detail the single photon channel for producing branons and KK gravitons at the LHC and their characteristic missing energy and transverse momentum signatures. In the first case, we need the cross section of the subprocess $q \bar{q} \rightarrow \gamma \pi \pi$, that was computed in [18]:

$$
\begin{aligned}
\frac{d \sigma(q \bar{q} \rightarrow \gamma \pi \pi)}{d k^{2} d t}= & \frac{Q_{q}^{2} \alpha N\left(k^{2}-4 M^{2}\right)^{2}}{184320 f^{8} \pi^{2} \hat{s}^{3} t u} \sqrt{1-\frac{4 M^{2}}{k^{2}}} \\
& \times\left(\hat{s} k^{2}+4 t u\right)\left(2 \hat{s} k^{2}+t^{2}+u^{2}\right),
\end{aligned}
$$

where $N$ is the number of branons (that we will assume degenerate and equal to the number of extra dimensions), $\hat{s} \equiv\left(p_{1}+p_{2}\right)^{2}, t \equiv\left(p_{1}-q\right)^{2}, u \equiv\left(p_{2}-q\right)^{2}$ and $k^{2} \equiv$ $\left(k_{1}+k_{2}\right)^{2} \cdot p_{1}$ and $p_{2}$ are the initial quark and antiquark four-momenta; $q$, the final photon four-momentum; and $k=k_{1}+k_{2}$, the total branon four-momentum. Thus, the contribution to the total cross section for the $p p \rightarrow \gamma \pi \pi$ reaction is

$$
\begin{aligned}
\sigma(p p \rightarrow \gamma \pi \pi)= & \int_{x_{\min }}^{1} d x \int_{y_{\min }}^{1} d y \sum_{q} \bar{q}_{p}(y ; \hat{s}) q_{p}(x ; \hat{s}) \\
& \times \int_{k_{\min }^{2}}^{k_{\max }^{2}} d k^{2} \int_{t_{\min }}^{t_{\max }} d t \frac{d \sigma(q q \rightarrow \gamma \pi \pi)}{d k^{2} d t} .
\end{aligned}
$$

Here $q_{p}(x ; \hat{s})$ and $\bar{q}_{p}(y ; \hat{s})$ are the quark and antiquark distribution functions of the proton; $x$ and $y$ are the fractions of the proton's energy carried by the initial quark and antiquark.

For the KK graviton analysis, as for the branon case, we need the cross section of the subprocess $q \bar{q} \rightarrow \sum_{n} \gamma h^{(n)}$, that was computed in [9]. It can be written as

$$
\begin{aligned}
& \frac{d \sigma\left(q \bar{q} \rightarrow \sum_{n} \gamma h^{(n)}\right)}{d m^{2} d t} \\
& =\frac{Q_{q}^{2} \alpha}{48 m^{2} M_{D}^{2} \hat{s}^{3} t u}\left(\frac{m^{2} \pi}{M_{D}^{2}}\right)^{N / 2}\left(\hat{s} m^{2}+4 t u\right)\left(2 \hat{s} m^{2}+t^{2}+u^{2}\right),
\end{aligned}
$$

where $N$ is the number of extra dimensions $(N=D-4)$, $M_{D}$ is associated with the fundamental gravitational scale in the $D$-dimensional bulk space, and we have approximated the KK masses by the continuous variable $m$.

\footnotetext{
${ }^{1}$ The proper ratio to be compared for the KK modes decoupling in flexible brane worlds is not $M_{D}$ vs $f$, but $\Lambda$ vs $f$, where $\Lambda$ is the cutoff of the effective theory associated with the KK gravitons. However, one can expect both scales $\left(M_{D}\right.$ and $\left.\Lambda\right)$ to be related. Please read [17] for further details.
}

We can see that the single photon cross sections for KK gravitons and for branons are very similar. The continuous KK mass plays the roll of the invariant mass of the branon pair. In fact, for $N=6$ and massless branons, the cross section is identical with the identification: $M_{10}^{8}=320 \pi^{5} f^{8}$ [19]. Moreover, independently of the number of dimensions and the branon mass, the angular dependence factorizes in the same way for branons and KK gravitons. Therefore, it seems difficult to distinguish between both signals by using a pseudorapidity analysis. The invariant mass study is more promising. On the one hand, the number of extra dimensions changes the power law dependence in the graviton case. Therefore, this analysis can exclude the branon explanation for a possible excess. On the other hand, a non-negligible branon mass, as it would be in the case of branon dark matter, introduces a lower cut in the signature that cannot be reproduced with the KK graviton tower.

\section{PYTHIA SIMULATIONS}

To simulate the effective vertex of our $2 \rightarrow 3$ processes and to estimate the experimental cuts used by the ATLAS Collaboration [20] we have used the general purpose event generator PYTHIA 8.175 [21] and its internal phase space selection machinery by inheriting the base class Sigma3Process [20]. We also have used the intrinsic random number generator included in PYTHIA 8 [22] which, according to PYTHIA's documentation [21], provides uniquely different random number sequences as long as the integer seeds remain below 900,000,000.

We do not consider CMS data [23] since ATLAS results are slightly more constraining. For example, the bound on $M_{D}$ for $N=6$ is $1.6 \mathrm{TeV}$ for CMS [23] and $1.9 \mathrm{TeV}$ for ATLAS [20]. A combined analysis can only improve the constraints we have obtained in this work. However, it is beyond the scope of our study, since we do not have access to the full information related to detector efficiencies and systematic uncertainties. In any case, the final results will not be modified importantly since the constraints to the KK graviton and branon models do not depend crucially on statistics. Such effective theories are more sensitive to the total energy of the process.

In both the branon and graviton cases, we have set $\sqrt{s}=7 \mathrm{TeV}$ and the same cuts to fit the conditions of [20]. The next conditions are required:

(i) One isolated photon with $p_{T}>150 \mathrm{GeV}$ (transverse momentum) and pseudorapidity $|\eta| \in[0,1.37) \cup$ (1.52, 2.37).

(ii) A number of jets less than or equal to 1 . The used clustering algorithm is the anti-kT one with an $R$ distance parameter $0.4 \mathrm{GeV}$, a minimum transverse momentum $p_{T}>30 \mathrm{GeV}$ and a maximum pseudorapidity $|\eta|<4$.5. Only observable final-state particles are included in the analysis. Both the high $p_{T}$ photon and the hypothetical dark matter (DM) 
particles are explicitly excluded. The true masses of particles are also used.

(iii) In a cone of $\Delta R=\sqrt{(\Delta \eta)^{2}+(\Delta \phi)^{2}}=0.4$ around the photon the sum of the energies of all the visible particles (excluding the DM particles) is less than $5 \mathrm{GeV}$.

(iv) A transverse missing momentum $E_{T}^{\text {miss }}>150 \mathrm{GeV}$. To compute it, we take into account all the visible particles with $|\eta|<4.9$.

(v) The reconstructed photon, transverse missing momentum and jet (if found) are separated by $\Delta \phi\left(\gamma, E_{T}^{\text {miss }}\right)>$ $0.4, \Delta R(\gamma$, jet $)>0.4$ and $\Delta \phi\left(\right.$ jet, $\left.E_{T}^{\text {miss }}\right)>0.4$.

(vi) There are no electrons, positrons or muons. This restriction applies to electrons (and positrons) with $p_{T}>20 \mathrm{GeV}$ and $|\eta|<2.47$, and to muons with $p_{t}>10 \mathrm{GeV}$ and $|\eta|<2.4$. However, in compliance with our simulations, the effect of this restriction over the signal is negligible although, according to Ref. [20], it is expected to reduce the background.

The internal machinery of PYTHIA 8 has been configured with the cuts (see Ref. [20])

(i) PhaseSpace: pTHatMin $=1 \mathrm{GeV}$

(ii) PhaseSpace: $\mathrm{pTHat} 3 \mathrm{Min}=1 \mathrm{GeV}$

(iii) PhaseSpace: pTHat5Min $=1 \mathrm{GeV}$

(iv) PhaseSpace: RsepMin $=0.1$

The first three set the invariant moment $p_{T}$ cut to $1 \mathrm{GeV}$. And the last one sets the minimum separation $\Delta R=\sqrt{(\Delta \eta)^{2}+(\Delta \phi)^{2}}$ between any two outgoing partons to $\Delta R>0.1$.

The speed of simulation varies, so for each value of $M$ (branons) or $N$ (gravitons) we have generated 100 histograms in $E_{T}^{\text {miss }}$ imposing that each execution take 10 hours. The variables $f$ (branons) and $M_{D}$ (gravitons), since they are a multiplicative factor in the differential cross section, are introduced through a rescaling of the histogram. The only simulated hard event is our effective vertex.

We have extracted the experimental data from Ref. [20], which corresponds to the ATLAS data of 2011, with $\sqrt{s}=$ $7 \mathrm{TeV}$ and an integrated luminosity of $4.6 \mathrm{fb}^{-1}$. Since the effect of an increase of both $f$ and $M_{D}$ is a decrease in the squared matrix element, a $\chi^{2}$ test is performed to find a lowest limit in both variables for each $M$ (or $N$ ). The experimental value of the number of detected events is taken as the measured events minus the background estimated by Ref. [20], and the variance $\sigma^{2}$ as $\sigma^{2}=\sigma_{\text {data }}^{2}+$ $\sigma_{\text {background }}^{2}$. A confidence limit of $95 \%$ has been used. This variance enters into the chi-squared analysis which allows us to set lowest limits over $M_{D}$ (for the graviton model) and $f$ (for branons).

\section{CONCLUSIONS}

We have obtained constraints for the branon model in the parameter space $(f, M)$ by using ATLAS data in the single-photon channel at $\sqrt{s}=7 \mathrm{TeV}$ for the first time. With the same data, we have tested our procedure by obtaining again constraints on the ADD KK graviton parameter space $\left(M_{D}, N\right)$. We have shown explicitly the improvement of these results by comparing with previous studies using the same channel from Tevatron and LEP.

Our analysis applies to the fundamental constants of the models commented above. It is interesting to note that KK gravitons and branons contribute also virtually. As it has been discussed independently for KK gravitons [24-27] and branons [5,17], there are important restrictions on these models by studying other channels, such as twophoton, lepton pair, top quark pair, and $e^{-} p$ production. However, these constraints are not related to $M_{D}$ (in the KK graviton case) or $f$ (in the case of branons). On the contrary, they allow us to constrain a combination of these fundamental scales and the cutoff of both effective theories. The question is whether these virtual contributions are divergent and in general, if they need to be regularized. Therefore, one can interpret these bounds as restrictions on new physics scales but not strictly on the fundamental couplings of the KK graviton or the branon field (read [17] for further details).

Our Monte Carlo computations for the two models of both branons and KK gravitons are shown in Fig. 1, along with the SM-background computation and the experimental points of the ATLAS Collaboration [20] $(\sqrt{s}=7 \mathrm{TeV}$ and $\int L d t=4.6 \mathrm{fb}^{-1}$ ). It can be seen that no signal of

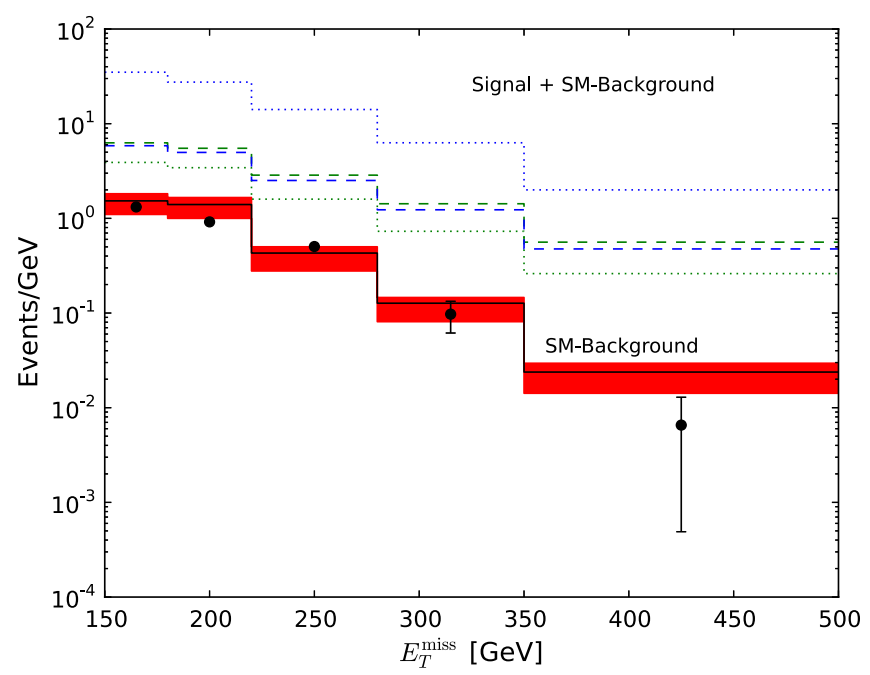

FIG. 1 (color online). The ATLAS $E_{T}^{\text {miss }}$ distribution (black dots, $\sqrt{s}=7 \mathrm{TeV}, \int L d t=4.6 \mathrm{fb}^{-1}$ ) versus the SM background (red band, see [20]) and our own computations for both the KK graviton + SM background (dashed lines) and the branon + SM background (dotted lines). The represented graviton models use $M_{D}=1 \mathrm{TeV}$ and $N=2$ (lower blue dashed line), and $M_{D}=1.5 \mathrm{TeV}$ and $N=6$ (upper green dashed line), and the branon one $M=2 \mathrm{TeV}, N=1$ and $f=60 \mathrm{GeV}$ (upper blue dotted line) and $M=1 \mathrm{TeV}, N=1$ and $f=200 \mathrm{GeV}$ (lower green dotted line). 
CEMBRANOS, DELGADO, AND DOBADO

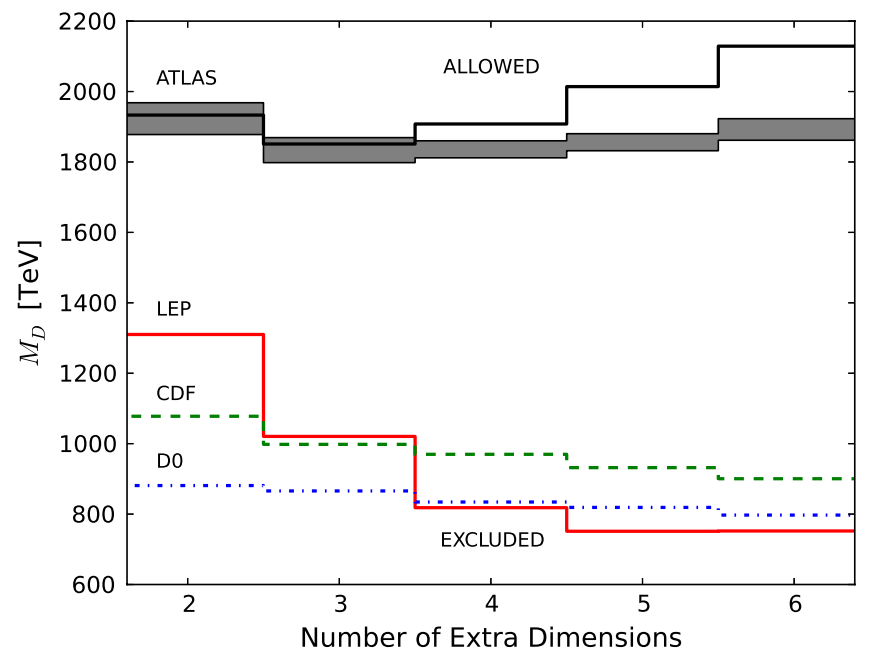

FIG. 2 (color online). Computed lowest limit (according to ATLAS data with $\sqrt{s}=7 \mathrm{TeV}$ and $\int L d t=4.6 \mathrm{fb}^{-1}$ ) for the value of $M_{D}$ parameter of the KK graviton model (black solid line), versus the NLO computation of Ref. [20] (gray band) and limits of LEP (solid red line), CDF (green dashed line) and D0 (blue dash dotted line).

either model is found, and the experimental points are compatible with the SM background.

Thus, the main goal of our computation is giving a lowest limit in the value of $f$ parameter of the branon model for various extra dimensions $N$ (see Fig. 3). In all cases, a confidence limit of $95 \%$ has been used. In order to test our model, we also compute the lowest limit in the value of $M_{D}$ parameter of the KK graviton model (Fig. 2) and compare it with the computation of Ref. [20].

According to Fig. 3, although the fit is good for low values of $N$, our limit is overestimated for high $N$ by a factor $\approx 15 \%$ in the worst case. However, it is remarkable that we are using a tree-level squared matrix element, while Ref. [20] uses a next-to-leading order (NLO) calculation and it has access to a full detector simulation. In any case,
PHYSICAL REVIEW D 88, 075021 (2013)

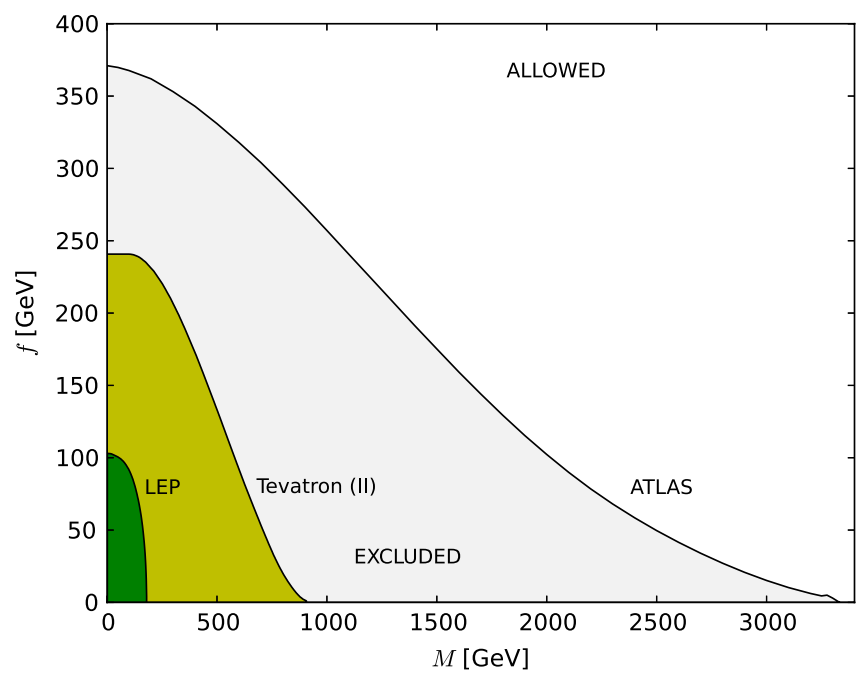

FIG. 3 (color online). Computed exclusion region (according to ATLAS data) for the value of $f$ parameter of the branon (gray area) versus the limits of the second run of Tevatron (dark yellow area, Ref. [18], $\sqrt{s}=1.96 \mathrm{TeV}$ and $\int L d t=200 \mathrm{pb}^{-1}$ ) and LEP (green area, Ref. [4], $\sqrt{s}=189-209 \mathrm{GeV}$ ).

our analysis provides the most constraining limits from collider experiments over the branon model.

\section{ACKNOWLEDGMENTS}

The authors thankfully acknowledge the computer resources, technical expertise, and assistance provided by the Barcelona Supercomputing Centre-Centro Nacional de Supercomputación - and the Tirant supercomputer support staff at Valencia. We are also pleased to thank Professor Antonio L. Maroto and Professor Torbjörn Sjöstrand for their useful help and comments. This work has been supported by MICINN (Spain) Project Nos. FIS 2008-01323, FIS2011-23000, and FPA2011-27853-01 and ConsoliderIngenio MULTIDARK CSD2009-00064. R. L. D. is a fellow of FPI, Ref. BES-2012-056054.
[1] A. Perez-Lorenzana, AIP Conf. Proc. 562, 53 (2001); V. A. Rubakov, Usp. Fiz. Nauk 171, 913 (2001) [Phys. Usp. 44, 871 (2001)]; Y. A. Kubyshin, arXiv:hep-ph/0111027; G. Gabadadze, arXiv:hep-ph/0308112; F. Feruglio, Eur. Phys. J. C 33, s114 (2004); C. Csaki, in Proceedings of Theoretical Advanced Study Institute, TASI 2002, Boulder, USA, 2002, edited by M. Shifman et al., From Fields to Strings Vol. 2, (2004) pp. 967-1060; arXiv:hep-ph/ 0404096; T. G. Rizzo, in The Proceedings of 32nd SLAC Summer Institute on Particle Physics (SSI 2004): Natures Greatest Puzzles, Menlo Park, California, 2004, pp. L013; G. Burdman, AIP Conf. Proc. 753, 390 (2005).
[2] V. A. Rubakov and M. E. Shaposhnikov, Phys. Lett. 125B, 136 (1983).

[3] N. Arkani-Hamed, S. Dimopoulos, and G. R. Dvali, Phys. Lett. B 429, 263 (1998); Phys. Rev. D 59, 086004 (1999); I. Antoniadis, N. Arkani-Hamed, S. Dimopoulos, and G. R. Dvali, Phys. Lett. B 436, 257 (1998).

[4] P. Achard et al., Phys. Lett. B 597, 145 (2004); S. Heinemeyer et al., arXiv:hep-ph/0511332; J.A. R. Cembranos, A. Rajaraman, and F. Takayama, arXiv:hepph/0512020; Europhys. Lett. 82, 21001 (2008); J. A. R. Cembranos, Hyperfine Interact. 215, 39 (2013); 215, 39 (2013); A. Juste et al., arXiv:hep-ph/0601112; ILC 
Collaboration, arXiv:0709.1893; ILC Collaboration, arXiv:0712.1950; ILC Collaboration, arXiv:0712.2356.

[5] S. C. Park and H. S. Song, Phys. Lett. B 523, 161 (2001); J. A. R. Cembranos, A. Dobado, and A. L. Maroto, Phys. Rev. D 73, 057303 (2006).

[6] J.A.R. Cembranos, J.L. Feng, A. Rajaraman, and F. Takayama, Phys. Rev. Lett. 95, 181301 (2005); J. A. R. Cembranos, J. L. Feng, and L. E. Strigari, Phys. Rev. D 75, 036004 (2007); Phys. Rev. Lett. 99, 191301 (2007); J. A. R. Cembranos and L. E. Strigari, Phys. Rev. D 77, 123519 (2008); J. A. R. Cembranos, Phys. Rev. Lett. 102, 141301 (2009); Phys. Rev. D 73, 064029 (2006).

[7] J. A. R. Cembranos, A. de la Cruz-Dombriz, A. Dobado, R. A. Lineros, and A. L. Maroto, Phys. Rev. D 83, 083507 (2011); Phys. Rev. D 83, 083507 (2011); J. A. R. Cembranos, A. de la Cruz-Dombriz, V. Gammaldi, and A. L. Maroto, Phys. Rev. D 85, 043505 (2012); J. A. R. Cembranos, V. Gammaldi, and A. L. Maroto, Phys. Rev. D 86, 103506 (2012); J. Cosmol. Astropart. Phys. 04 (2013) 051; J. A. R. Cembranos, A. Cruz-Dombriz, V. Gammaldi, R. A. Lineros, and A. L. Maroto, J. High Energy Phys. 09 (2013) 077.

[8] T. Biswas, J. A. R. Cembranos, and J. I. Kapusta, Phys. Rev. Lett. 104, 021601 (2010); J. High Energy Phys. 10 (2010) 048; Phys. Rev. D 82, 085028 (2010); J. A. R Cembranos, K. A. Olive, M. Peloso, and J.-Philippe Uzan, J. Cosmol. Astropart. Phys. 07 (2009) 025; J. A. R. Cembranos, C. Hallabrin, A. L. Maroto, and S. J. Núñez Jareño, Phys. Rev. D 86, 021301 (2012); J. A. R. Cembranos, A. L. Maroto, and S. J. Núñez Jareño, Phys. Rev. D 87, 043523 (2013); F. D Albareti, J.A.R Cembranos, and A. de la Cruz-Dombriz, J. Cosmol. Astropart. Phys. 12 (2012) 020; F. D Albareti, J.A. R Cembranos, A. de la Cruz-Dombriz, and A Dobado, J. Cosmol. Astropart. Phys. 07 (2013) 009; J. A. R. Cembranos, Phys. Rev. D 73, 064029 (2006); J. A. R Cembranos, A. de la Cruz-Dombriz, and B. M. Núñez, J. Cosmol. Astropart. Phys. 04 (2012) 021; A. de la CruzDombriz, and D. Sáez-Gómez, Entropy 14, 1717 (2012).

[9] G. F. Giudice, R. Rattazzi, and J.D. Wells, Nucl. Phys. B544, 3 (1999).
[10] R. Sundrum, Phys. Rev. D 59, 085009 (1999); T. Kugo and K. Yoshioka, Nucl. Phys. B594, 301 (2001).

[11] A. Dobado and A. L. Maroto, Nucl. Phys. B592, 203 (2000).

[12] J. A. R. Cembranos, A. Dobado, and A. L. Maroto, Phys. Rev. Lett. 90, 241301 (2003); Phys. Rev. D 68, 103505 (2003).

[13] J. A. R. Cembranos, A. Dobado, and A. L. Maroto, Phys. Rev. D 65, 026005 (2001); J. Phys. A 40, 6631 (2007).

[14] J. Alcaraz, J. Cembranos, A. Dobado, and A. Maroto, Phys. Rev. D 67, 075010 (2003).

[15] J. A. R. Cembranos, A. Dobado, and A. L. Maroto, Int. J. Mod. Phys. D 13, 2275 (2004); A. L. Maroto, Phys. Rev. D 69, 043509 (2004); 69, 101304 (2004); J. A. R. Cembranos, A. de la Cruz-Dombriz, A. Dobado, and A. L. Maroto, J. Cosmol. Astropart. Phys. 10 (2008) 039.

[16] M. Bando, T. Kugo, T. Noguchi, and K. Yoshioka, Phys. Rev. Lett. 83, 3601 (1999).

[17] J. A. R. Cembranos, A. Dobado, and A. L. Maroto, Phys. Rev. D 73, 035008 (2006).

[18] J.A. R. Cembranos, A. Dobado, and A.L. Maroto, Phys. Rev. D 70, 096001 (2004); J. A. R. Cembranos, J. L. Diaz-Cruz, and L. Prado, Phys. Rev. D 84, 083522 (2011).

[19] P. Creminelli and A. Strumia, Nucl. Phys. B596, 125 (2001).

[20] G. Aad and T. Abajyan (ATLAS Collaboration), Phys. Rev. Lett. 110, 011802 (2013).

[21] T. Sjöstrand, S. Mrenna, and P.Z. Skands, J. High Energy Phys. 05 (2006) 026; Comput. Phys. Commun. 178, 852 (2008).

[22] G. Marsaglia, A. Zaman, and W.-W. Tsang, Stat. Probab. Lett. 9, 35 (1990).

[23] S. Chatrchyan et al. (CMS Collaboration), Phys. Rev. Lett. 108, 261803 (2012).

[24] J. L. Hewett, Phys. Rev. Lett. 82, 4765 (1999).

[25] G. F. Giudice, R. Rattazzi, and J.D. Wells, Nucl. Phys. B544, 3 (1999).

[26] K. Agashe and N. G. Deshpande, Phys. Lett. B 456, 60 (1999).

[27] M. Marionneau (ATLAS and CMS Collaborations), arXiv:1305.3169. 